Supplement of

\title{
Building the bridge between safety requirements and numerical modeling: an example considering crack development of Opalinus clay in laboratory and field scales
}

Tuanny Cajuhi et al.

Correspondence to: Tuanny Cajuhi (tuanny.cajuhi@bgr.de)

The copyright of individual parts of the supplement might differ from the article licence. 
Building the bridge between safety requirements and numerical modeling: An example considering crack development of Opalinus Clay in laboratory and field scales 


\section{Motivation \& Aims}

- Motivation

- Radioactive waste needs to be stored in a safe and sustainable manner

- The integrity of the rock, i.e. its containment capabilities, must be ensured

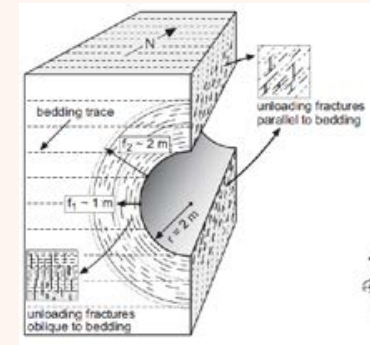

Conceptual model extracted from [Tsang et al. 2012]

- Understanding the coupled phenomena taking place in the rock needs, ideally, to occur at the in-situ scale

- Aims

- Correlate process understanding in the near field and its impact on the integrity of the containment providing rock zone (CRZ)

- Illustrate an example for the role of numerical modeling in safety assessment 


\section{Scope}

- "The term integrity describes the conservation of the properties related to the confining capacity of the containment providing rock zone of a repository"

„Der Begriff Integrität beschreibt den Erhalt der Eigenschaften des Einschlussvermögens des einschlusswirksamen Gebirgsbereichs eines Endlagers“ [BMU 2020]

- What can affect the integrity? For how long?

- Which processes are correlated to a possible integrity loss?

\section{Construction/ \\ ventilation}

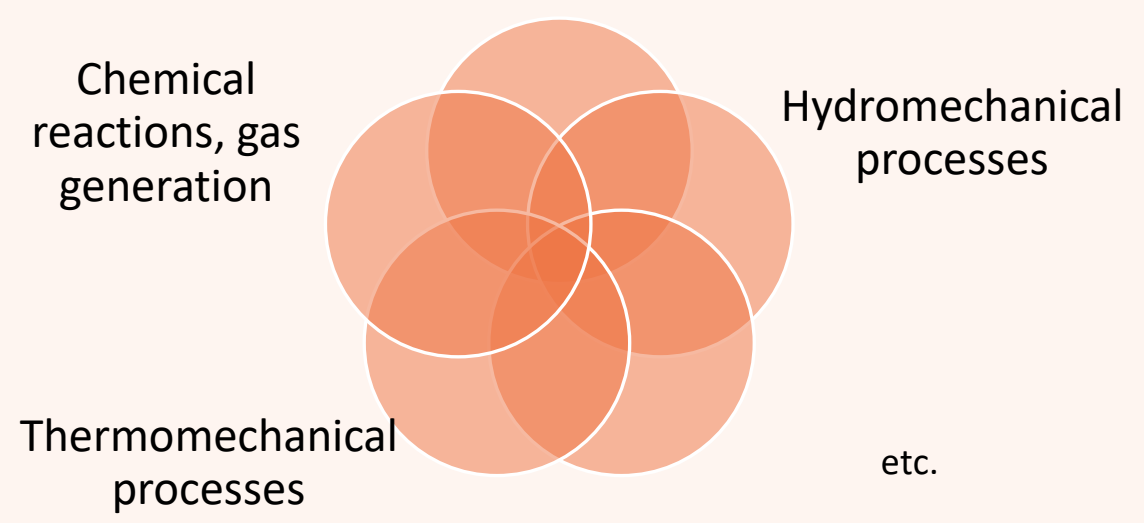




\section{In the field}

\section{- Initial condition}

- Excavation

- Stress redistribution

- Plastic behavior (shear / compressive strength criterion)

- EDZ development (cracks, permeability increase)
- Functional period

- Wetting and drying (seasonal changes)

- Further degradation and/or increase of EDZ

- Cracks due to drying
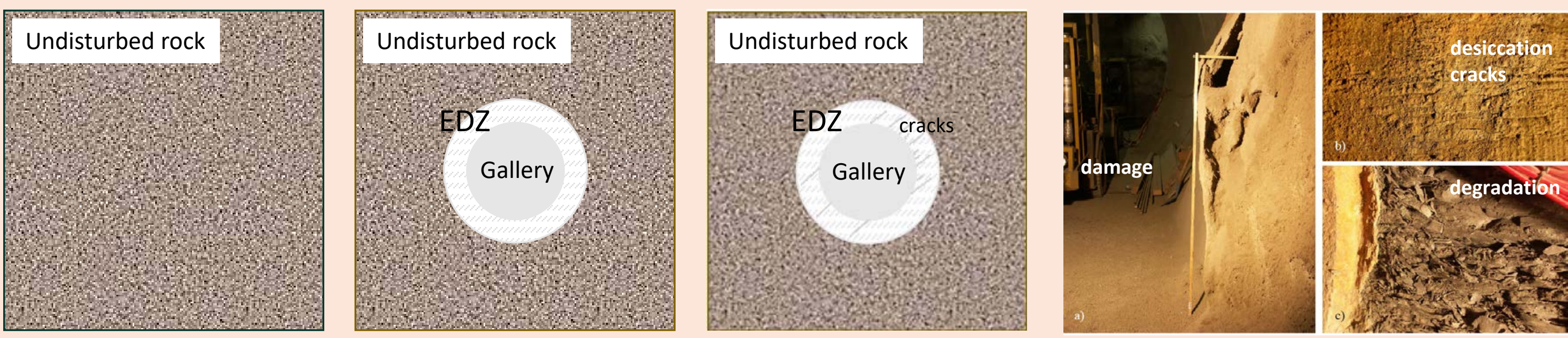

OPA [Technischer Bericht 14-02 Nagra] 


\section{Practical steps}

\section{Formulation}

- The "host" rock

- Mont Terri Rock Laboratory

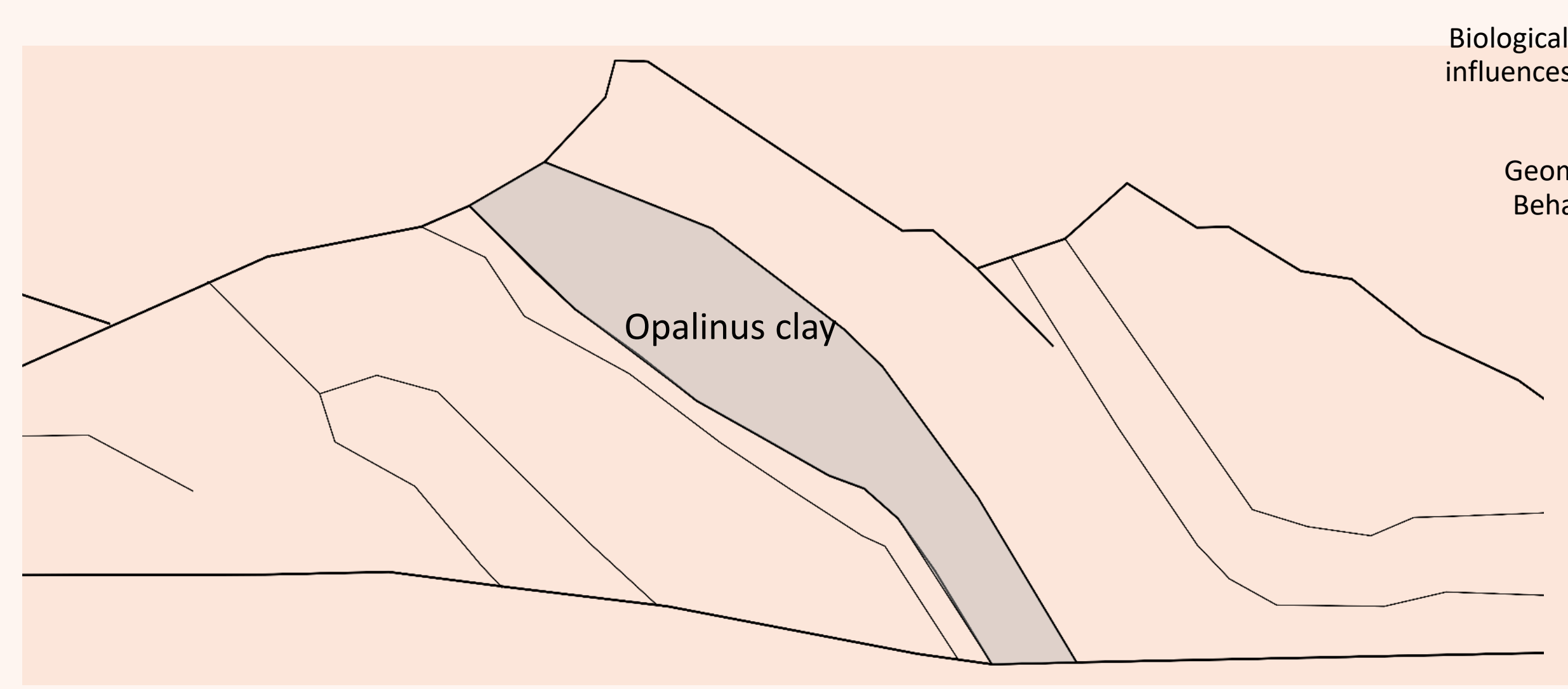

In-situ

conditions

Heterogeneities

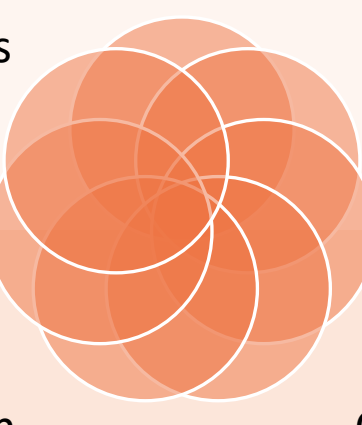

eomech. Behavior
Temperature

Hydraulic behavior

Coupled processes
Interdisziplinäres

Forschungssymposium
für die Sicherheit der

für die Sicherheit der
nuklearen Entsorgung

What are the points we would like to tackle? 


\section{Practical steps}

\section{Simplification}

म?

Interdisziplinäres

Forschungssymposium
für die Sicherheit der nuklearen Entsorgung

- Cyclic-Deformation (CD-A) experiment: free twin niches

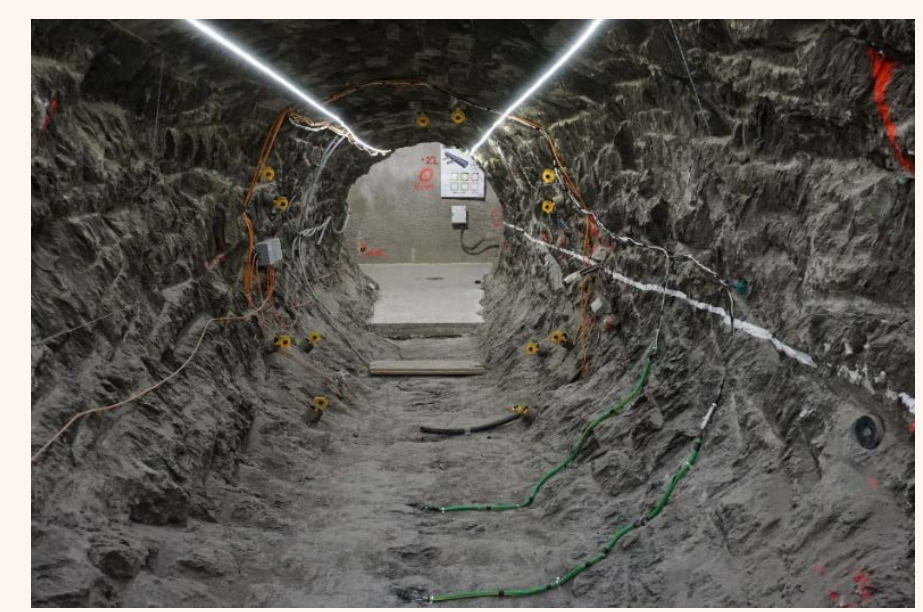

Open niche

- Seasonal humidity variations

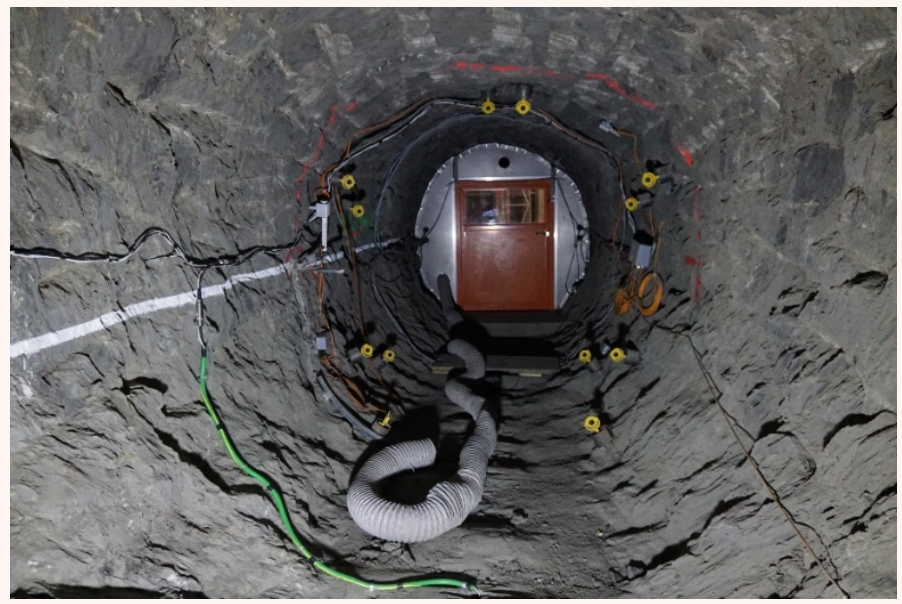

Closed niche

- Controlled high humidity
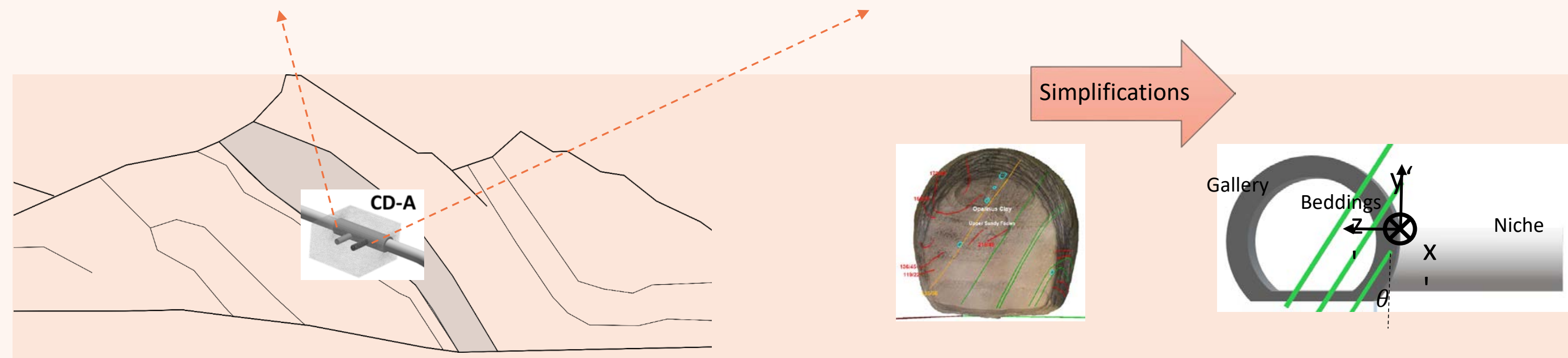


\section{Practical steps}

\section{Mathematical modeling}

- Hydro-mechanical (HM) framework

- Balance equations

$$
\begin{aligned}
& \nabla \cdot\left(\mathrm{nS}_{\mathrm{l}} \mathbf{v}_{\mathrm{ls}}\right)+\rho_{\mathrm{l}}\left[\mathrm{C}_{\mathrm{s}} \frac{\partial \mathrm{p}_{\mathrm{l}}}{\partial \mathrm{t}}+\mathrm{S}_{\mathrm{l}} \alpha \frac{\partial \operatorname{tr}\left(\nabla^{\mathrm{s}} \mathbf{u}\right)}{\partial \mathrm{t}}\right]=0 \\
& \nabla \cdot\left(\boldsymbol{\sigma}^{\prime}-\alpha \mathrm{S}_{\mathrm{l}} \mathrm{p}_{\mathrm{l}} \mathbf{I}\right)+\mathrm{nS}_{\mathrm{l}} \rho_{\mathrm{l}} \mathbf{g}+(1-\mathrm{n}) \rho_{\mathrm{s}} \mathbf{g}=\mathbf{0}
\end{aligned}
$$

- Constitutive relations

- Transversely isotropic, linear elastic material

- Van Genuchten fitting

- Initial conditions

$$
\begin{gathered}
\mathrm{p}_{\mathrm{w}}=\mathrm{p}_{\mathrm{w}_{0}} \text { at } \mathrm{t}=0 \\
\mathbf{u}=\mathbf{u}_{0} \text { at } \mathrm{t}=0 \\
\sigma_{0} \text { at } \mathrm{t}=0
\end{gathered}
$$

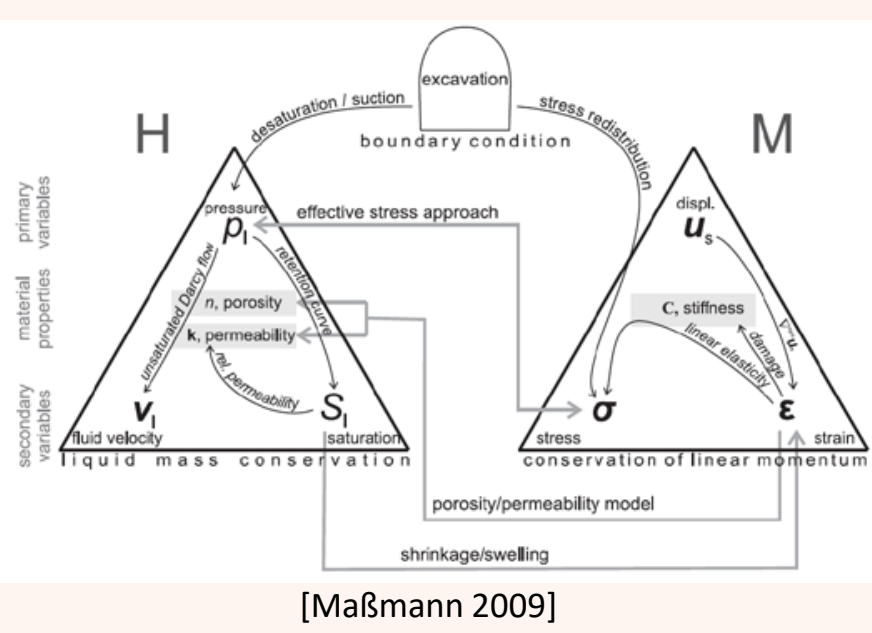

- Capillary pressure (Richards assumption)

$$
\begin{gathered}
\mathrm{p}_{\mathrm{c}}=\mathrm{p}_{\mathrm{g}}-\mathrm{p}_{\mathrm{l}} \\
\mathrm{p}_{\mathrm{g}}=\mathrm{p}_{\mathrm{atm}}
\end{gathered}
$$

- Rate of liquid flow (Darcy's law)

$$
\mathrm{nS}_{\mathrm{l}} \mathbf{v}_{\mathrm{ls}}=-\rho_{\mathrm{l}} \mathrm{k}_{\mathrm{rl}} \frac{\mathbf{K}_{\mathbf{i}}}{\mu_{\mathrm{l}}}\left(\nabla \mathrm{p}_{\mathrm{l}}-\rho_{\mathrm{l}} \mathbf{g}\right)
$$

- Boundary conditions

$$
\begin{gathered}
\mathbf{u}=\overline{\mathbf{u}} \text { on } \Gamma_{\mathrm{u}} \\
\boldsymbol{\sigma} \cdot \mathbf{n}=\overline{\mathbf{t}} \text { on } \Gamma_{\mathrm{t}} \\
\mathrm{p}_{\mathrm{w}}=\overline{\mathrm{p}}_{\mathrm{l}} \text { on } \Gamma_{\mathrm{p}} \\
\overline{\mathrm{q}} \text { on } \Gamma_{\mathrm{q}}
\end{gathered}
$$

OpenGeoSys 


\section{Practical steps}

Numerical modeling
$\mathbf{B C P}$ Bundesanstat tir

Geowissenschaften und Rohstoffe

GEOZENTRUM HANNOVER
मी?

Interdisziplinäres

Forschungssymposium

ür die Sicherheit der

- Design

- First predictions using independent (in-/output) data from the experiment

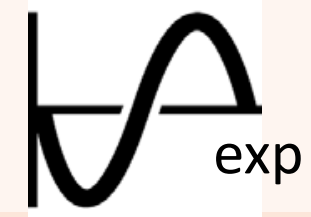

IC

$\sigma_{\text {in-situ }}$

$p_{\text {in-situ }}$
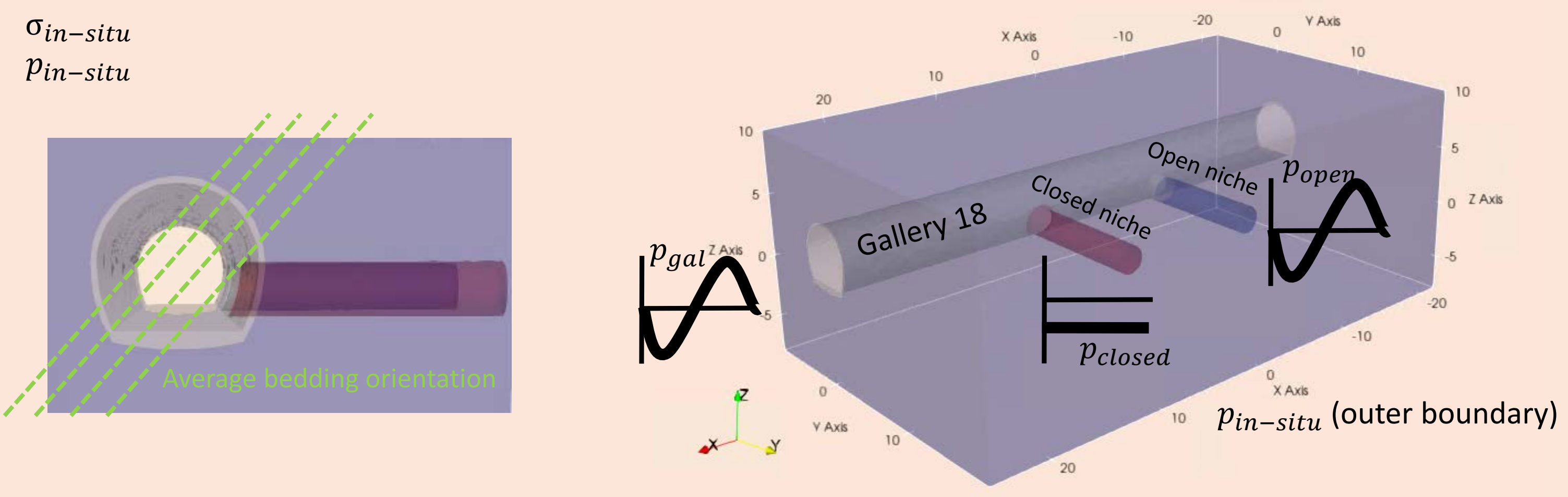


\section{Practical steps \\ Numerical modeling}

- Initial conditions and influence of excavation

- Hydro-mechanical behavior (long-term)

- Comparison with experiments within interdisciplinary approach Ziefle et al. 2021 (under review)

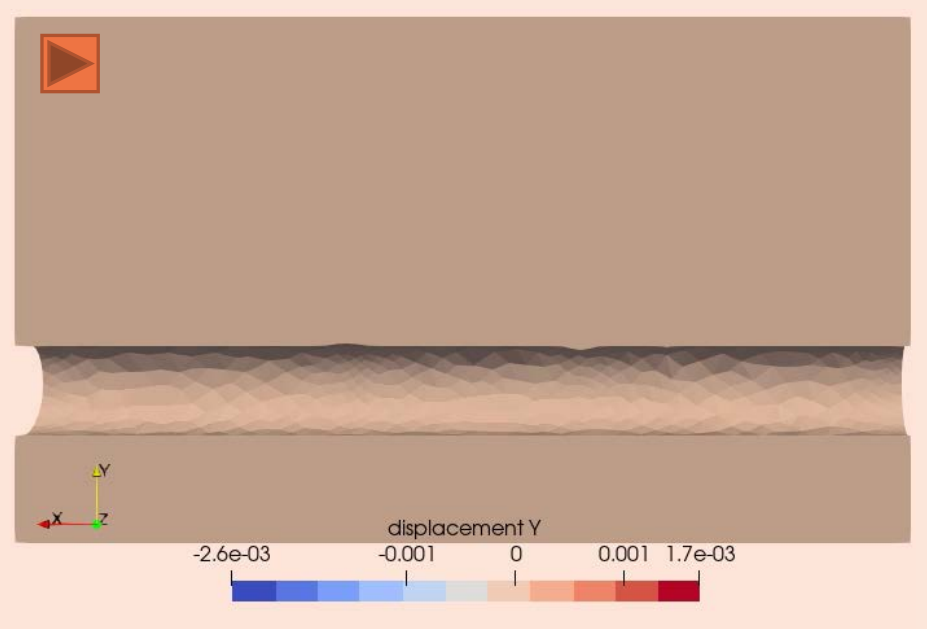

Displacement during excavation (HM)

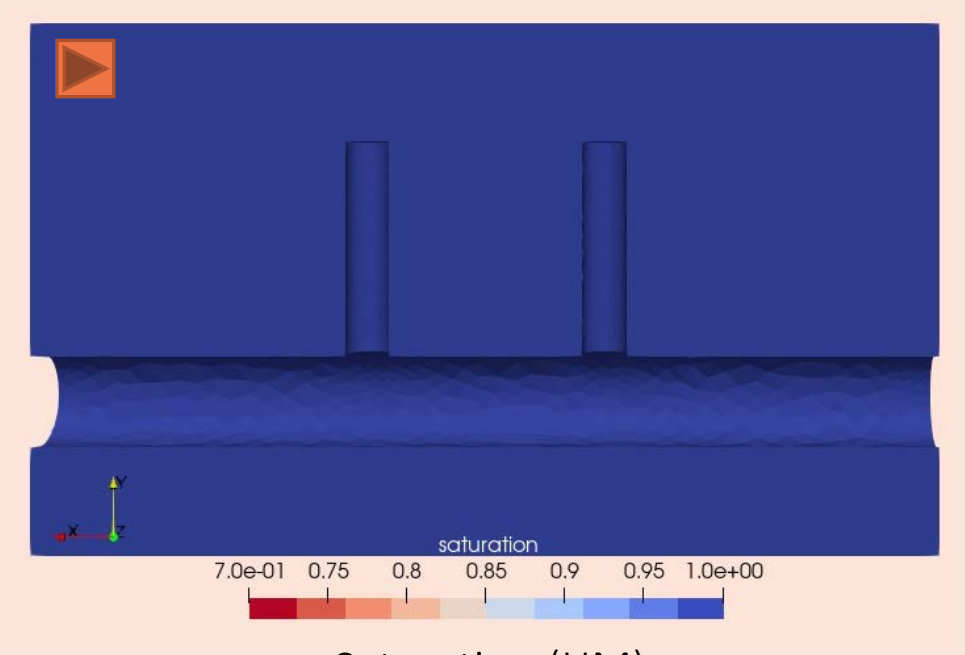

Saturation (HM)

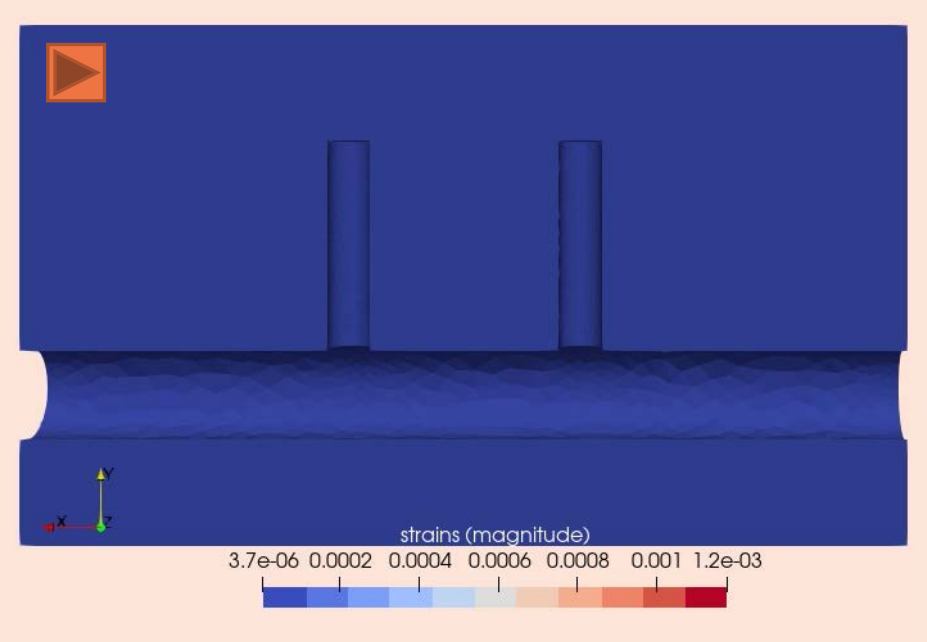

Strains (HM)
- Integrity?

- How far is the near field?

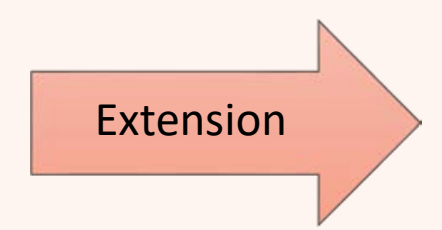

Interdisziplinäres

Forschungssymposium

nuklearen Entsorgung

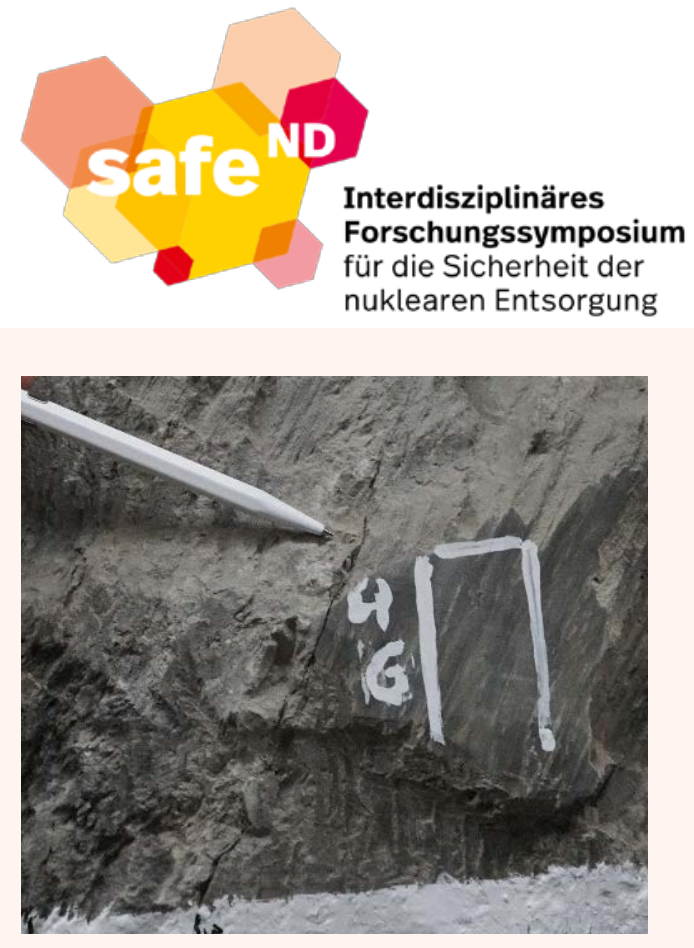

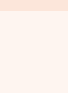




\section{Practical steps \\ Model extension}

- Identification of the mechanisms at the field and laboratory scales: shrinkage-swelling, cracking

- Application of the method in the smaller scale: assessment of numerical problems

- Adaptation and verification at larger scale
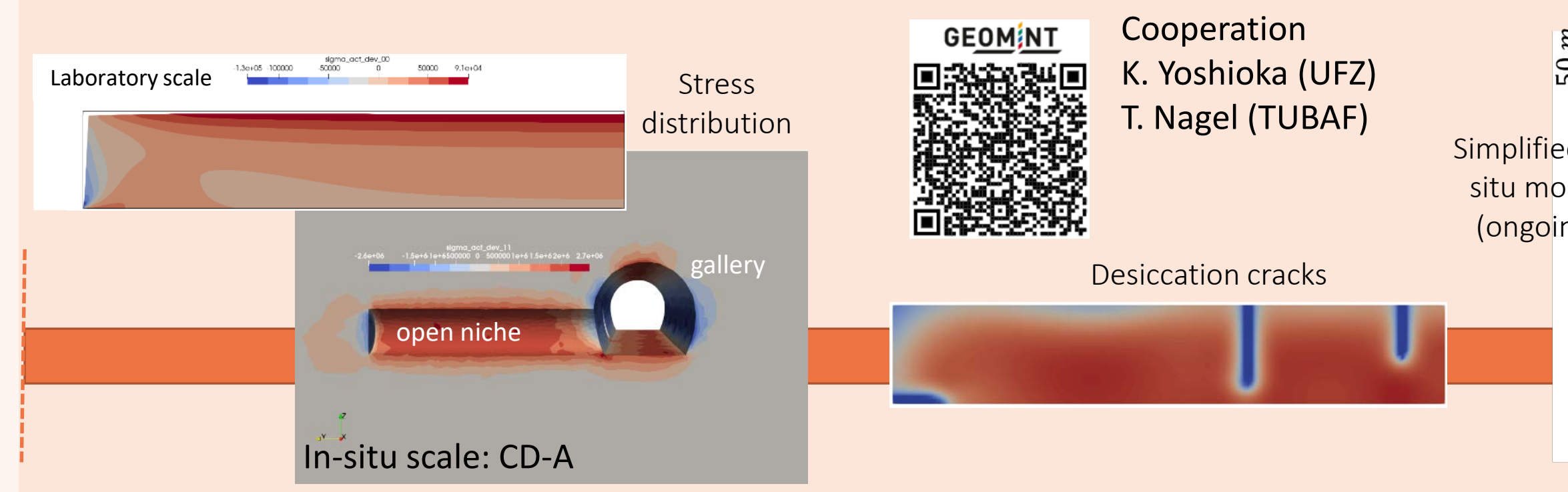

Cooperation

K. Yoshioka (UFZ)

T. Nagel (TUBAF)

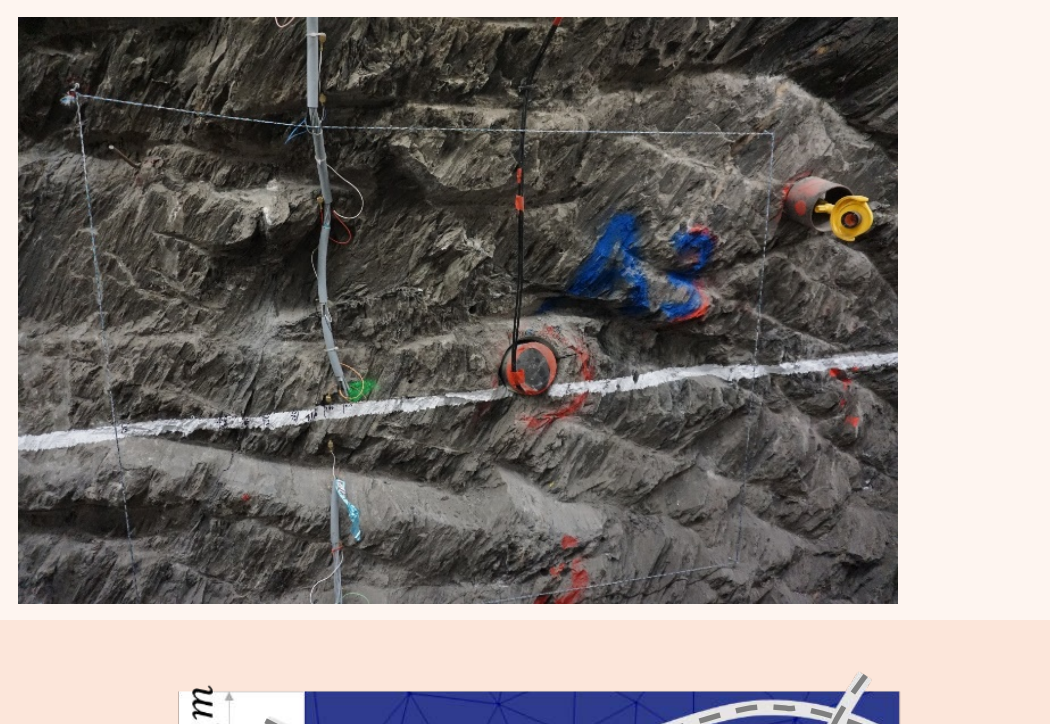

Extension of HM model with phase-field framework to account for crack evolution

mplified insitu model ongoing)

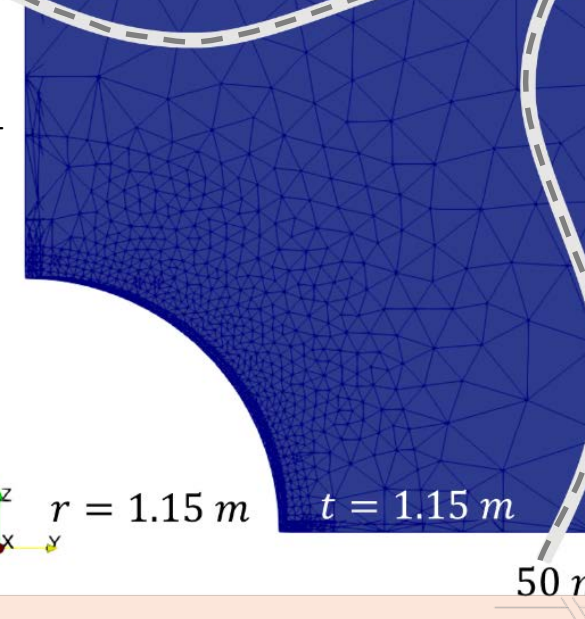




\section{Conclusions \& Outlook}

- Correlate process understanding and integrity requirements

- Practical steps on numerical modeling

- CD-A experiment, Mont Terri

- Use of experimental data

- Simulation of excavation and long-term behavior

- Further development and application of numerical methods

- Identification of mechanisms at laboratory and field scales

- Concept and perspectives for modeling cracking

- Transfer between the scales 